\title{
Computation of Flow over a Rotating Body on Unstructured Chimera Mesh
}

\author{
X. Zhang*, G. W. He, S. Z. Ni \\ LNM, Institute of Mechanics, Chinese Academy of Sciences, Beijing 100080, China \\ Email: zhangx@lnm.imech.ac.cn
}

\begin{abstract}
Flow around moving boundary is ubiquitous in engineering applications. To increse the efficienly of the algorithm to handle moving boundaries is still a major challenge in Computational Fluid Dynamics (CFD). The Chimera grid method is one type of method to handle moving boundaries. A concept of domain de-composition has been proposed in this paper. In this method, sub-domains are meshed independently and governing equations are also solved separately on them. The Chimera grid method was originally used only on structured (curvilinear) meshes. However, in a problem which involves both moving boundary and complex geometry, the number of sub-domains required in a traditional (structured) Chimera method becomes fairly large. Thus the time required in the interior boundary locating, link-building and data exchanging also increases. The use of unstructured Chimera grid can reduce the time consumption significantly by the reduction of domain(block) number.

Generally speaking, unstructured Chimera grid method has not been developed. In this paper, a wellknown pressure correction scheme - SIMPLEC is modified and implemented on unstructured Chimera mesh. A new interpolation scheme regarding the pressure correction is proposed to prevent the possible decoupling of pressure. A moving-mesh finite volume approach is implemented in an inertial reference frame. This approach is then used to compute incompressible flow around a rotating circular and elliptic cylinder. These numerical examples demonstrate the capability of the proposed scheme in handling moving boundaries. The numerical results are in good agreement with other experimental and computational data in literature. The method proposed in this paper can be efficiently applied to more challenge cases such as free-falling objects or heavy particles in fluid.
\end{abstract}

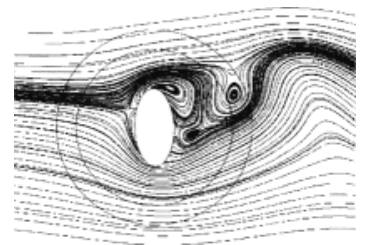

Figure: One snapshot of instantaneous streamlines of the flow over a rotating elliptic cylinder

\section{REFERENCES}

1. Maruoka A. Finite element analysis for flow around a rotating body using Chimera method. Int $J$ Comput Fluid Dynamics, 2003; 17(4): 289-97

2. Demirdzic I, Muzaferija S. Numerical method for coupled fluid flow, heat transfer and stress analysis using unstructured moving meshes with cells of arbitrary topology. Comput Meth Appl Mech Eng, 1995; 125: 235-55

3. Zhang $X$. Computation of viscous incompressible flow using pressure correction method on unstructured Chimera grid. Int J Comput Fluid Dynamics, 2007; (In Press). DOI: 10.1080/10618560601140094 